\title{
THUMPD3-ASI Is Correlated With Non-Small Cell Lung Cancer And Regulates Self-Renewal Through miR-543 And ONECUT2
}

This article was published in the following Dove Press journal: OncoTargets and Therapy

\author{
Jia Hu \\ Youfang Chen \\ Xiaodong $\mathrm{Li}$ \\ Huikai Miao \\ Rongzhen Li \\ Dongni Chen \\ Zhesheng Wen
}

Department of Thoracic Oncology, State Key Laboratory of Oncology in South China, Collaborative Innovation Center for Cancer Medicine, Sun Yat-Sen University Cancer Center, Guangzhou 510060, People's Republic of China
Correspondence: Zhesheng Wen Email wenzhsh@sysucc.org.cn
Background: Of all malignancies, lung cancer is the leading cause of death, and non-small cell lung cancer (NSCLC) accounts for $80-85 \%$ of all lung cancers. In this study, the long non-coding RNA (lncRNA) THUMPD3-AS1 was observed to be highly expressed in NSCLC and correlated with TNM stages and relapse, suggesting that THUMPD3-AS1 is involved in the regulation of NSCLC.

Methods: The aim of this study was to investigate the regulatory function and mechanism of THUMPD3-AS1 in NSCLC cells by cellular function and molecular biology experiments. Results: Overexpression and knockdown analysis revealed that THUMPD3-AS1 promoted tumor progression by increasing cell proliferation and self-renewal of NSCLC cells. Moreover, THUMPD3-AS1 may act as an endogenous sponge of microRNA-543 (miR543) which can regulate the target gene ONECUT2 in NSCLC cells.

Conclusion: Our study indicated that THUMPD3-AS1 regulated NSCLC cell self-renewal by regulating the expression of miR-543 and ONECUT2, and THUMPD3-AS1 can potentially act as a biomarker or therapeutic target in NSCLC.

Keywords: non-small cell lung cancer, self-renewal, THUMPD3-AS1, miR-543, ONECUT2

\section{Introduction}

Lung cancer is one of the most serious malignant tumors threatening human health. In the past 50 years, due to growing tobacco consumption and serious environmental pollution, lung cancer, which has the highest morbidity and mortality worldwide, has become one of the major diseases to seriously endanger human health. ${ }^{1-3}$ According to its histological characteristics, lung cancer can be divided into four types: adenocarcinoma, squamous cell carcinoma, large cell carcinoma and small cell carcinoma. Of these, adenocarcinoma, squamous cell carcinoma and large cell carcinoma have similar biological behavior, gene mutation and treatment options, and they are classified as non-small cell lung cancer (NSCLC), accounting for $80-85 \%$ of all lung cancers. ${ }^{4}$ As signs are lacking in the early stage of tumor onset, most NSCLC patients are diagnosed at an advanced stage or have progressed when diagnosed with specific symptoms. ${ }^{5}$

Long non-coding RNA (lncRNA) is a functional RNA molecule whose transcript length exceeds 200 nucleotides. ${ }^{6}$ LncRNA, which lacks the ability to encode proteins and is located in the nucleus or cytoplasm, can regulate gene expression at various levels, such as epigenetics, transcriptional regulation and post-transcriptional 
regulation. ${ }^{7-9}$ LncRNA can be divided into sense lncRNA, antisense lncRNA, bidirectional lncRNA, intragenic lncRNA and intergenic lncRNA, and have different roles in regulating cell functions, including cell proliferation, differentiation and development. ${ }^{10,11}$ Recently, more and more studies have shown that IncRNA plays an important role in the occurrence and development of cancer due to its transcription abnormalities, epigenetic modification abnormalities or chromosomal abnormalities. ${ }^{12,13}$ In NSCLC, it was also found that lncRNA was associated with poor tumor prognosis, and a number of lncRNAs have been found to affect the development of NSCLC as oncogenes or tumor suppressor genes. ${ }^{14,15}$

It was reported that lncRNA THUMPD3-AS1 (THUMPD3 antisense RNA 1) was upregulated in lung cancer; however, the regulatory mechanism of THUMPD3-AS1 is not yet clear. ${ }^{16}$ In this study, we found that THUMPD3-AS1 was upregulated in primary NSCLC tissues compared with adjacent NSCLC tissues. In addition, high expression levels of THUMPD3-AS1 were significantly associated with advanced TNM stages and relapse in NSCLC patients. Furthermore, we investigated the biological functions of THUMPD3-AS1 and found that THUMPD3-AS1 affected cell proliferation and selfrenewal of NSCLC cells. Mechanistically, we found that THUMPD3-AS1 acted as an endogenous sponge of microRNA-543 (miR-543) to regulate the expression of ONECUT2 (one cut homeobox 2), which affected cell proliferation and self-renewal. Thus, our results demonstrated that THUMPD3-AS1 was associated with NSCLC and involved in the regulation of self-renewal through miR-543 and ONECUT2.

\section{Methods}

\section{Cell Culture And Transfection}

NSCLC epithelial cell lines (A549 and H1299 cells, purchased commercially (Shanghai Biotechnology Corporation), ATCC cell lines) were cultured in RPMI (Roswell Park Memorial Institute) 1640 medium (Gibco, USA) supplemented with $10 \%$ fetal bovine serum at $37^{\circ} \mathrm{C}$ in a humidified atmosphere with $5 \% \mathrm{CO}_{2}$. The expression plasmids of THUMPD3-AS1 and ONECUT2 were constructed by inserting cDNA into the pCDNA3.1 plasmid. The shRNA plasmid of THUMPD3-AS1 was designed for lentivirus production. The siRNA of ONECUT2 designed for transfection. shRNA lentiviral plasmids were transfected into cells by lentivirus. The miRNA mimics,
miRNA inhibitors, siRNAs and plasmids were transfected into cells using Lipofectamine 2000 (Invitrogen, USA).

\section{Tissue Sample Collection}

NSCLC tissues, which were obtained by aspiration biopsy and immediately snap-frozen in liquid nitrogen and stored at $-80^{\circ} \mathrm{C}$ until use, were collected from the Sun Yat-sen University Cancer Center, and the following criteria were met: patients with primary NSCLC; a histological diagnosis of NSCLC with at least one measurable lesion; a TNM clinical stage of I to IV. All patients provided written informed consent, and the collection of NSCLC tissues for research purposes was approved by the relevant human research ethics committees of the Sun Yat-sen University Cancer Center. Patient characteristics are shown in Table 1.

\section{RNA Extraction And RT-PCR}

Total RNA of cells and tissue samples were extracted by Trizol (Invitrogen, USA). The fractions of cell nucleus and cytoplasm were extracted using PARIS Kit (Life Technologies, USA). Reverse transcription polymerase chain reaction (RT-PCR) was performed by using ABI ViiATM7Dx Real-Time PCR System (Life Technologies, USA). For lncRNA and mRNA detection, $1 \mu \mathrm{g}$ total RNA

Table I Overall Patient Characteristics

\begin{tabular}{|l|l|}
\hline Clinicopathological Factors & Number (\%)/Median (IQR) \\
\hline $\begin{array}{l}\text { Total: } \\
\mathrm{N}\end{array}$ & $5 \mathrm{I}$ \\
\hline $\begin{array}{l}\text { Histological type: } \\
\text { Adenocarcinoma } \\
\text { Squamouscarcinoma }\end{array}$ & $33(64.7 \%)$ \\
\hline $\begin{array}{l}\text { Gender: } \\
\text { Male }\end{array}$ & $18(35.3 \%)$ \\
Female & $36(70.6 \%)$ \\
\hline Age (years): & $15(29.4 \%)$ \\
Mean & $56.6(25-73)$ \\
\hline Smoking & \\
Nonsmoker & $20(39.2 \%)$ \\
Smoker & $31(60.8 \%)$ \\
\hline TNM Clinical stage, $\mathbf{n}(\%)$ & $7(13.7 \%)$ \\
I & $19(33.3 \%)$ \\
II & $8(15.7 \%)$ \\
II & \\
\hline
\end{tabular}


was used for cDNA synthesis using a Reverse Transcription Kit (Takara, Japan), then cDNA was used for RT-PCR using primers and TB Green Realtime PCR Kit (Takara, Japan). For miRNA detection, $1 \mu \mathrm{g}$ total RNA was used for cDNA synthesis using a miScript II RT Kit (Qiagen, Germany), then cDNA was used for RT-PCR using miScript Primer Assay and miScript SYBR Green PCR Kit (Qiagen, Germany). Expression levels of IncRNA, mRNA and miRNA were normalized by GAPDH and U6 separately.

The RT-PCR primers were designed as the following:

THUMPD3-AS1: Forward 5'-TGGTGCCACCTATGT TGTGG-3'; Reverse 5'-ACACTTCAGCCAGCAGAGA C-3'.

ONECUT2: Forward 5'-ATGCGAGTTCAGATGCTG CT-3'; Reverse 5'-CACCGGGGCTTGGACTAAAT-3'.

GAPDH: Forward 5'-GAAAGCCTGCCGGTGACTA A-3'; Reverse 5'-TTCCCGTTCTCAGCCTTGAC-3'.

\section{RNA Pull-Down Assay}

Biotinylated miR-543 probes (wild type bio-miR-543-WT and mutant type bio-miR-543-MUT) were incubated with strepavidin magnetic beads at $4{ }^{\circ} \mathrm{C}$ over-night to generate probe-coated magnetic beads. The cells were harvested and lysed by RIPA buffer. Cell lysates were incubated with probe-coated beads for $1 \mathrm{~h}$ at room temperature, and the isolated complexes were used for extraction of the RNA for RT-PCR analysis.

\section{Dual-Luciferase Reporter Assay}

The wild type (WT) and mutant type (MUT) 3'UTR of ONECUT2 and THUMPD3-AS1 were chemically synthesized and inserted into pGL3 plasmids (Promega, USA) for getting the wild type (ONECUT2-3'UTR-WT, THUMPD3-AS1-WT) or mutant type (ONECUT23'UTR-MUT, THUMPD3-AS1-MUT). 50 ng plasmids were transfected in combination with $1.5 \mathrm{nmol}$ of the miRNA mimics using Lipofectamine Transfection Reagent (Invitrogen, USA). Luciferase activity was measured $48 \mathrm{~h}$ after transfection using the Dual Luciferase Reporter Assay System (Promega, USA). The relative luciferase activity (firefly luciferase) was normalized to pRL-TK activity (Renilla luciferase).

\section{Western Blotting}

The cells were harvested and lysed by RIPA (Radio Immunoprecipitation Assay) buffer. $50 \mu \mathrm{g}$ heat-denatured proteins were loaded into a $15 \%$ sodium dodecyl sulfate- polyacrylamide gel electrophoresis (SDS-PAGE) system, and then transferred to a polyvinylidene difluoride membrane for Western blotting analysis. After blocking nonspecific binding sites with 5\% (wt/vol) non-fat milk, $0.1 \%$ (vol/vol) Tween-20 diluted in Tris ( $\mathrm{pH} 7.8$ )-buffered saline, rabbit polyclonal anti-ONECUT2 (Abcam, USA, 1:400 dilution) and anti-GAPDH (Abcam, USA, 1:1000 dilution) primary antibodies were added and incubated for $2 \mathrm{~h}$ at $37^{\circ} \mathrm{C}$. Then, HRP (horseradish peroxidase) conjugated goat anti-rabbit secondary antibody (Abcam, USA, 1:2000 dilution) was added and incubated for $2 \mathrm{~h}$.

\section{Cell Growth Assay}

Cell growth was determined at 1, 2, 3, 4, 5 days using Cell Counting Kit-8 (Beyotime, China). NSCLC cells were incubated in $90 \mu \mathrm{l}$ of RPMI-1640 medium and $10 \mu \mathrm{l}$ of CCK8 solution at $37{ }^{\circ} \mathrm{C}$ until visual color conversion occurred. The absorbance at $450 \mathrm{~nm}$ wavelength was measured on an automated reader.

\section{Colony Formation Assay}

NSCLC cells were trypsinized and plated to assess clonogenic survival (1000 cells per well in 6-well plates). Cells were allowed to form colonies over 7 days. The cells were then stained with GIEMSA (Sigma-Aldrich, USA) and counted using ImageJ software.

\section{Sphere Formation Assay}

NSCLC cells were plated in DMEM (Dulbecco's Modified Eagle Medium) F12 serum-free media (Gibco, USA) reconstituted with $20 \mathrm{ng} / \mathrm{mL}$ of EGF, $20 \mathrm{ng} / \mathrm{mL}$ of $\mathrm{bFGF}$, $2 \% \mathrm{~B} 27$, and $1 \%$ methylcellulose (5000 cells per well in a six-well plate). After 4-7 days, microsphere-like structures were visible, and images of the microspheres were captured using a microscope.

\section{Statistical Analysis}

All values (continuous variables and normally distributed) were expressed as mean \pm standard deviation (SD) from at least three separate experiments. The Student's unpaired $t$-test and chi-square test were performed using SPSS 21.0 statistical software. A two-tailed $P$ value test was used in all analyses, and differences were considered statistically significant if the $P$ value was less than $0.05(P<0.05)$. 


\section{Results}

\section{THUMPD3-ASI Is Upregulated In} NSCLC Tissues And Promotes SelfRenewal Of NSCLC Cells

In order to analyze the clinical correlations of THUMPD3-AS1 in NSCLC, the expression status of THUMPD3-AS1 in 51 pairs of primary and adjacent NSCLC samples was assessed by RT-PCR. As shown in Figure 1A, the expression of THUMPD3-AS1 in primary tissues (Cancer, C) was higher than that in adjacent tissues (Non-cancer, NC) $(P=0.012)$. We then analyzed the correlation between the expression level of THUMPD3-AS1 and the clinicopathologic features of NSCLC patients. As shown in Table 2, NSCLC patients with a high expression level of THUMPD3AS1 were more likely to have advanced TNM stages $(P=0.016)$ and relapse $(P=0.028)$ compared with NSCLC patients with a low expression level. Moreover, the expression level of THUMPD3-AS1 was not different due to histological type, gender, age and smoking history. Therefore, THUMPD3-AS1 was abnormally upregulated in NSCLC tissues and the high expression level of THUMPD3-AS1 was associated with increased tumor progression of NSCLC.

To investigate the roles of THUMPD3-AS1 in NSCLC cells, we used cDNA plasmid or short hairpin RNA (shRNA) to achieve overexpression or knockdown of THUMPD3-AS1 in A549 and H1299 cells (Figure 1B). First, we analyzed the effect of THUMPD3-AS1 on the growth of NSCLC cells, and found that overexpression of THUMPD3-AS1 promoted and knockdown of THUMPD3-AS1 inhibited growth in A549 and H1299 cells (Figure 1C). According to the colony formation assay results, overexpression of THUMPD3-AS1 promoted and knockdown of THUMPD3-AS1 inhibited colony formation capacity in A549 and H1299 cells (Figure 1D). Moreover, we examined the effect of THUMPD3-AS1 on the self-renewal of cells using the sphere formation assay. The results showed that overexpression of THUMPD3-AS1 increased and knockdown of THUMPD3-AS1 decreased the size and number of spheres (Figure 1E). Therefore, these results suggested that upregulation of THUMPD3-AS1 influenced NSCLC by promoting the proliferation and selfrenewal capability of NSCLC cells.

\section{THUMPD3-ASI Interacts With miR-543}

In order to elucidate the molecular mechanism involved in the control of NSCLC cell proliferation and self-renewal by THUMPD3-AS1, we found that THUMPD3-AS1 was predominantly located in the cytoplasm (Figure 2A). The recent competing endogenous RNA (ceRNA) hypothesis proposes that IncRNAs located in the cytoplasm can exert their function by sponging miRNAs, and we found that THUMPD3AS1 contained a putative binding site for miR-543 using the online predictive tools of bioinformatics (Figure 2B). We then analyzed the effect of THUMPD3-AS1 on miR-543 expression in A549 and H1299 cells, and found that overexpression of THUMPD3-AS1 decreased and knockdown of THUMPD3-AS1 increased the expression of miR-543 (Figure 2C). According to the results of the dual-luciferase reporter assay, miR-543 reduced the luciferase activity of the THUMPD3-AS1-WT reporter vector, and the inhibitory effect was abolished when the miR-543 binding site was mutated (THUMPD3-AS1-MUT) (Figure 2D). Moreover, in order to confirm the direct binding between THUMPD3AS1 and miR-543, we performed RNA pull-down assay using biotinylated miR-543. The results showed that THUMPD3-AS1 was more enriched in the biotinylated wild-type miR-543 (bio-miR-543-WT) captured fraction than in the biotinylated binding site mutant type miR-543 (bio-miR-543-MUT) (Figure 2E). These results suggested that THUMPD3-AS1 could function as a ceRNA by interacting with miR-543 in NSCLC.

\section{The miR-543 Regulates ONECUT2}

As miRNAs function by silencing target genes, we predicted the targets of miR-543 using the picTar and TargetScan prediction systems, and found that the $3^{\prime}$ untranslated region (3'UTR) of ONECUT2 contained a miR-543 binding site (Figure 3A). As shown in Figure 3B, it was observed that the protein and mRNA expression of ONECUT2 decreased after transfection with miR-543 mimics and increased after transfection with miR-543 inhibitors in A549 and H1299 cells (Figure 3B). Moreover, using the dual-luciferase reporter assay, it was found that miR-543 mimics decreased the relative luciferase activity by binding to the target site in the wild-type 3'UTR of ONECUT2 (ONECUT2-3'UTR-WT) compared with the binding site mutant type $3^{\prime} \mathrm{UTR}$ of ONECUT2 (ONECUT2-3'UTR-MUT) (Figure 3C). Therefore, these results suggested that ONECUT2 was the direct target gene of miR-543 in NSCLC. 
A

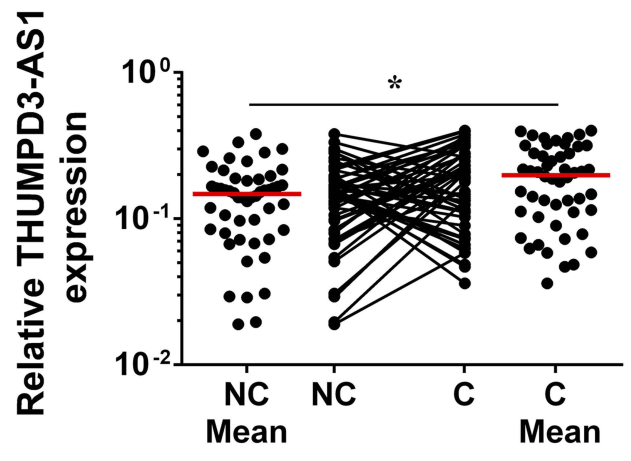

C

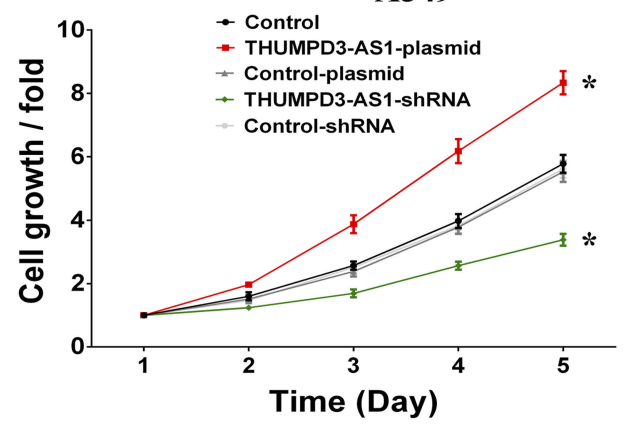

B

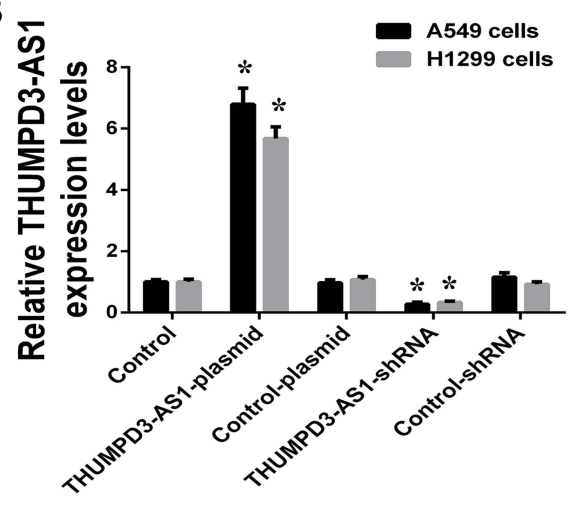

H1299

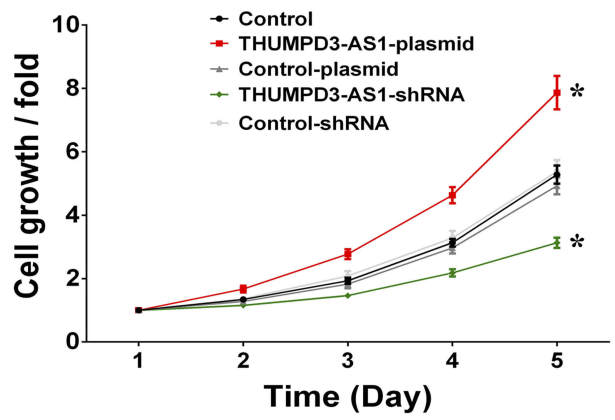

D

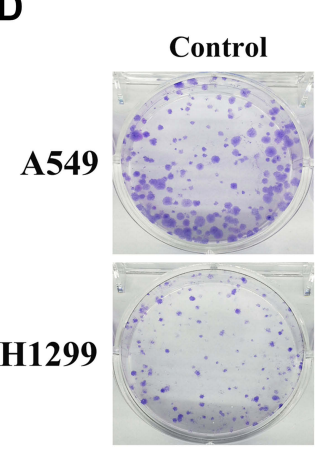

THUMPD3-AS1 THUMPD3-AS1
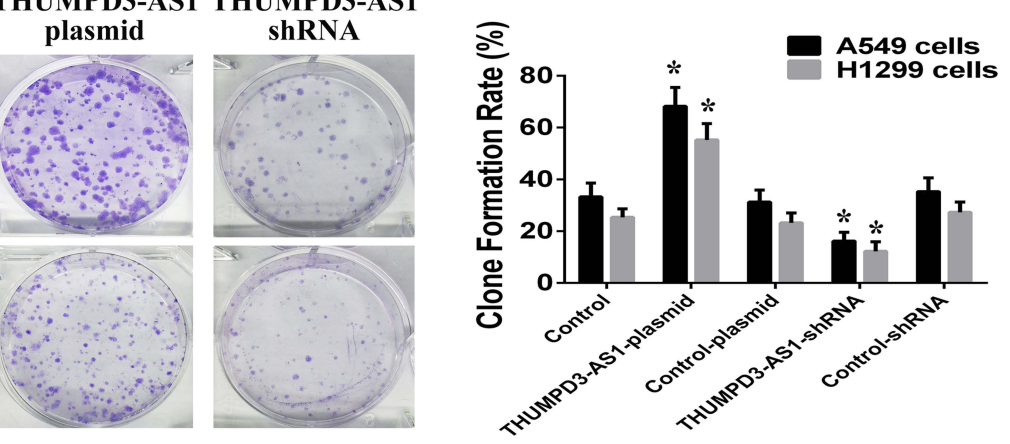

E

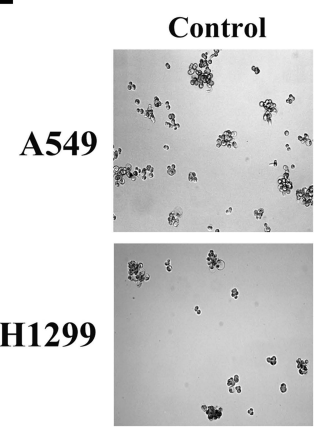

THUMPD3-AS1 THUMPD3-AS1
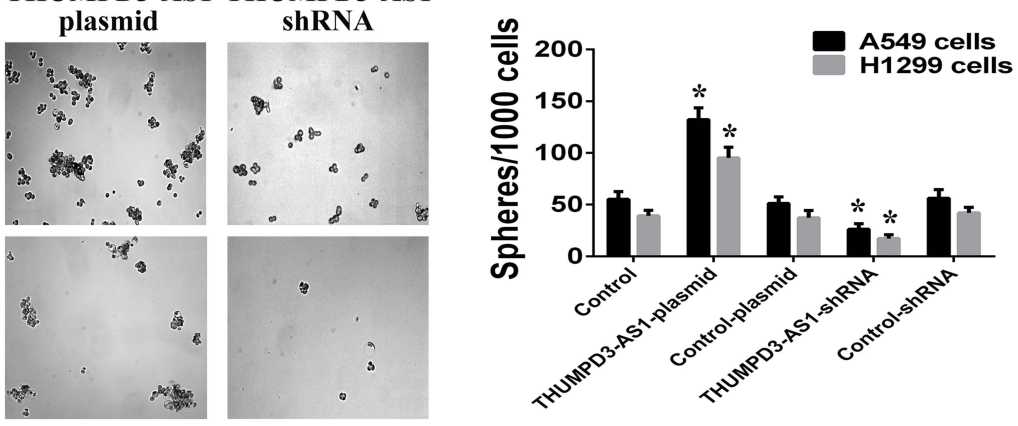

Figure I THUMPD3-ASI was abnormal upregulated in NSCLC and involved in the regulation of cell proliferation and self-renewal. (A) Scatter plots of expression levels of THUMPD3-ASI in NC and C tissues of NSCLC. NC, adjacent carcinoma tissues; C, primary carcinoma tissues. (B) THUMPD3-ASI expressions were increased after transfection of THUMPD3-ASI plasmids and decreased after transfection of THUMPD3-ASI shRNAs in A549 and HI299 cells. (C) Cell growth assay was used to observe the regulation effect of THUMPD3-ASI on the cell growth in A549 and HI299 cells. (D) Colony formation assay was used to observe the regulation effect of THUMPD3ASI on the colony-formation capability in A549 and HI299 cells. (E) Sphere formation assay was used to observe the regulation effect of THUMPD3-ASI on the size and number of spheres in A549 and HI299 cells. ( $* P<0.05$, error bar refers to SD). 
Table 2 Correlation Between The Expression Level Of THUMPD3-ASI And The Clinicopathological Features Of NSCLC Patients

\begin{tabular}{|c|c|c|c|c|c|}
\hline \multirow{2}{*}{\multicolumn{2}{|c|}{ Clinicopathological Factors }} & \multicolumn{2}{|c|}{$\begin{array}{l}\text { Expression Level }^{\mathrm{a}} \text { Of } \\
\text { THUMPD3-AS I }\end{array}$} & \multirow{3}{*}{$\begin{array}{l}\chi^{2} \text { value }\left(\text { Bold values } \chi^{2}>7.815(v=3)\right. \\
\left.\chi^{2}>3.84 I(v=1)\right) \\
0.202\end{array}$} & \multirow{3}{*}{$\begin{array}{l}P \text { value }(B o l d \\
\text { values } P<0.05) \\
0.653\end{array}$} \\
\hline & & \multirow{2}{*}{$\begin{array}{l}\text { High } \\
18 \\
11\end{array}$} & \multirow{2}{*}{$\begin{array}{l}\text { Low } \\
15 \\
7\end{array}$} & & \\
\hline $\begin{array}{l}\text { Histological } \\
\text { type }\end{array}$ & $\begin{array}{l}\text { Adenocarcinoma } \\
\text { Squamouscarcinoma }\end{array}$ & & & & \\
\hline Gender & $\begin{array}{l}\text { Male } \\
\text { Female }\end{array}$ & $\begin{array}{l}20 \\
9\end{array}$ & $\begin{array}{l}16 \\
6\end{array}$ & 0.085 & 0.771 \\
\hline Age (years) & $\begin{array}{l}<60 \\
\geq 60\end{array}$ & $\begin{array}{l}21 \\
8\end{array}$ & $\begin{array}{l}13 \\
9\end{array}$ & 1.003 & 0.317 \\
\hline Smoking & $\begin{array}{l}\text { Nonsmoker } \\
\text { Smoker }\end{array}$ & $\begin{array}{l}10 \\
19\end{array}$ & $\begin{array}{l}10 \\
12\end{array}$ & 0.629 & 0.428 \\
\hline $\begin{array}{l}\text { TNM Clinical } \\
\text { stage }\end{array}$ & $\begin{array}{l}\text { I } \\
\text { II } \\
\text { III } \\
\text { IV }\end{array}$ & $\begin{array}{l}2 \\
6 \\
15 \\
6\end{array}$ & $\begin{array}{l}5 \\
11 \\
4 \\
2\end{array}$ & 10.37 & 0.016 \\
\hline Relapse & $\begin{array}{l}0 \\
1\end{array}$ & $\begin{array}{l}6 \\
23\end{array}$ & $\begin{array}{l}11 \\
11\end{array}$ & 4.845 & 0.028 \\
\hline
\end{tabular}

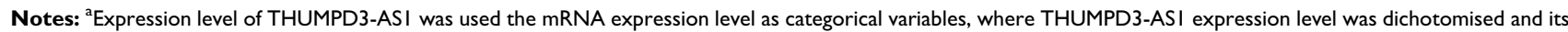
categories represented as follows: cases with higher expression in primary tissues compared with adjacent tissues were designated as "high", whereas cases with lower expression in primary tissues compared with adjacent tissues were designated as "low".

\section{THUMPD3-ASI Regulates ONECUT2 By Sponging miR-543}

As THUMPD3-AS1 shares the regulatory miR-543 with ONECUT2, we determined whether THUMPD3AS1 regulated ONECUT2 expression. In A549 and H1299 cells, overexpression of THUMPD3-AS1 increased ONECUT2 expression, while ONECUT2 expression recovered following co-transfection with miR-543 mimics and THUMPD3-AS1 plasmids (Figure 4A). Moreover, knockdown of THUMPD3AS1 decreased ONECUT2 expression, while ONECUT2 expression recovered following co-transfection with miR-543 inhibitors and THUMPD3-AS1 shRNAs (Figure 4A). In order to further determine whether THUMPD3-AS1 protected ONECUT2 from miR-543-mediated repression, we performed dual-luciferase reporter assays and co-transfected ONECUT23'UTR-WT and miR-543 mimics in the presence of THUMPD3-AS1 plasmids or control plasmids, respectively. We found that overexpression of THUMPD3-AS1 efficiently abolished the miR-543 induced reduction in luciferase activity of ONECUT23'UTR-WT (Figure 4B). These results indicated that THUMPD3-AS1 may act as an endogenous sponge of miR-543; thus, regulating ONECUT2 indirectly.

\section{THUMPD3-ASI Regulates Self-Renewal Through miR-543 And ONECUT2}

Finally, we investigated whether THUMPD3-AS1 regulated self-renewal through miR-543 and ONECUT2. According to the results of the cell growth, colony formation and sphere formation assays, the inhibitory effect of THUMPD3-AS1 knockdown on cell growth, proliferation and self-renewal recovered after transfection with ONECUT2 plasmids or miR-543 inhibitors in A549 and H1299 cells (Figure 5A, B and C). Moreover, the recovery effect of miR-543 inhibitors on cell growth, proliferation and self-renewal was abolished after transfection with ONECUT2 siRNAs in A549 and H1299 cells (Figure 5A, B and C). In conclusion, these results demonstrated that THUMPD3-AS1 regulates cell proliferation and self-renewal by regulating the expression of miR-543 and ONECUT2 in NSCLC.

\section{Discussion}

Recently, the natural miRNA sponge hypothesis suggested that there were endogenous miRNA sponges in organisms that negatively regulate miRNA, and ceRNA (competing endogenous RNA) is one of them. ${ }^{17}$ ceRNA is an endogenous RNA transcript that can compete with other RNA transcripts for the same miRNAs. ${ }^{18}$ The 
A

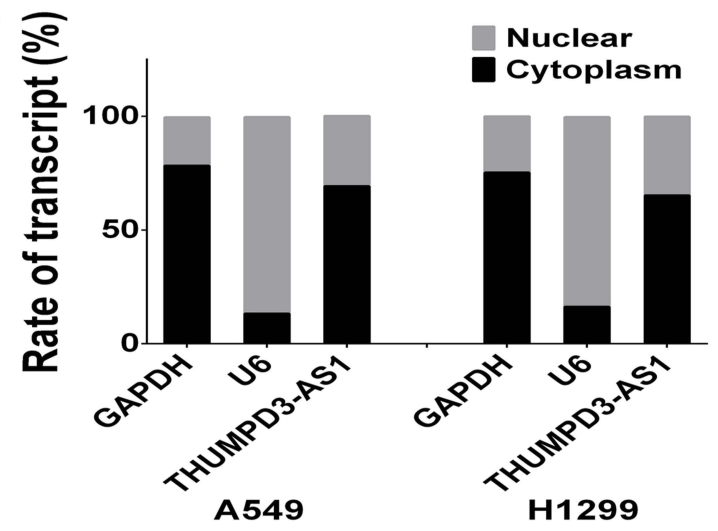

C

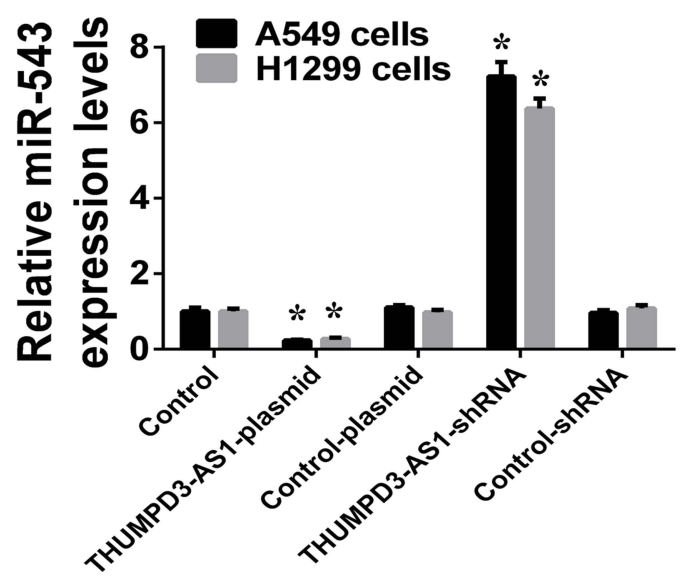

B

\author{
THUMPD3-AS1-WT \\ 5'-GUAUCCUCCAUU-UGAAUGUUG-3' \\ | | | | | || | \\ miR-543 3'-UUCUUCACGUGGCGCUUACAAA-5' \\ | | | \\ 5'-GUAUCCUCCAUU-UCUUACAAG-3' \\ THUMPD3-AS1-MUT
}

D

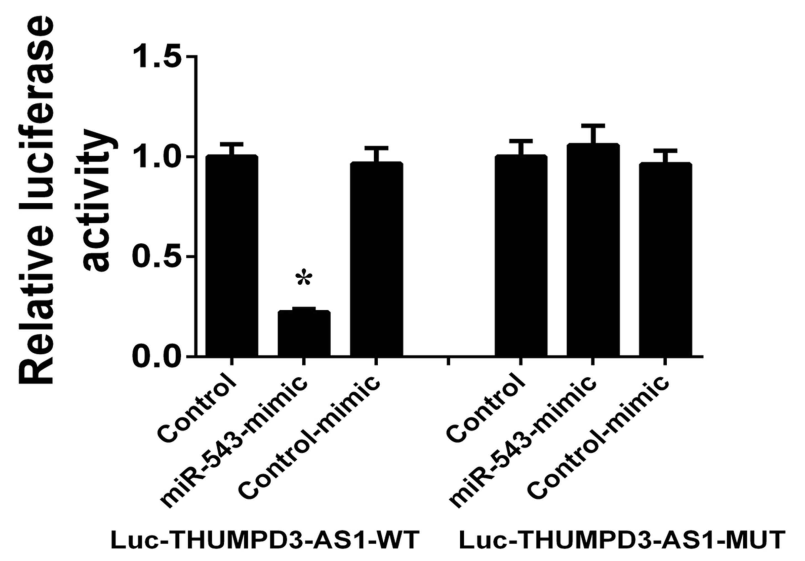

E

bio-miR-543-WT

3'-UUCUUCACGUGGCGCUUACAAA-5'

THUMPD3-AS1 5'-GUAUCCUCCAUU-UGAAUGUUG-3'

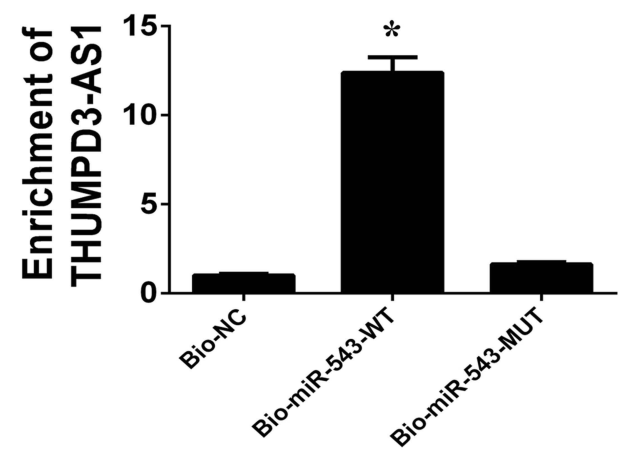

Figure 2 THUMPD3-ASI function as a ceRNA by interacting with miR-543. (A) The subcellular location of THUMPD3-ASI was tested in A549 and HI299 cells which were fractionated into cytoplasmic and nuclear fractions. U6 and GAPDH served as nucleus and cytoplasm control, respectively. (B) THUMPD3-ASI contains the binding site for miR543 , including wild type and mutant type. (C) THUMPD3-ASI regulated the expression of miR-543 in A549 and HI299 cells. (D) Luciferase reporter assay was used to observe the binding effect of miR-543 on the THUMPD3-ASI. (E) RNA pull-down assay was used to observe the direct binding between THUMPD3-ASI and miR-543. (*P < 0.05, error bar refers to SD).

ceRNA is the mode of "RNA regulate miRNA," which is complementary to the traditional mode of "miRNA regulate RNA," thus achieving mutual communication and regulation. ${ }^{19}$ ceRNAs compete to bind to miRNAs through miRNA responsive elements and regulate the target gene of miRNA by antagonizing the binding of miRNA with the $3^{\prime}$ UTR of the target gene, thus reducing repression of the target gene. ${ }^{20}$ It has also been found that disruption of ceRNA expression was involved in the promotion of cancer including NSCLC. ${ }^{21-23}$ In this 
A

ONECUT2-3'UTR-WT

5'-AGUAGCUACAUCAUGAAUGUUA-3'

miR-543 3'-UUCUUCACGUGGCGCUUACAAA-5'

| | | | |

5'-AGUAGCUACAUCAUCUUACAAA-3'

ONECUT2-3'UTR-MUT

C

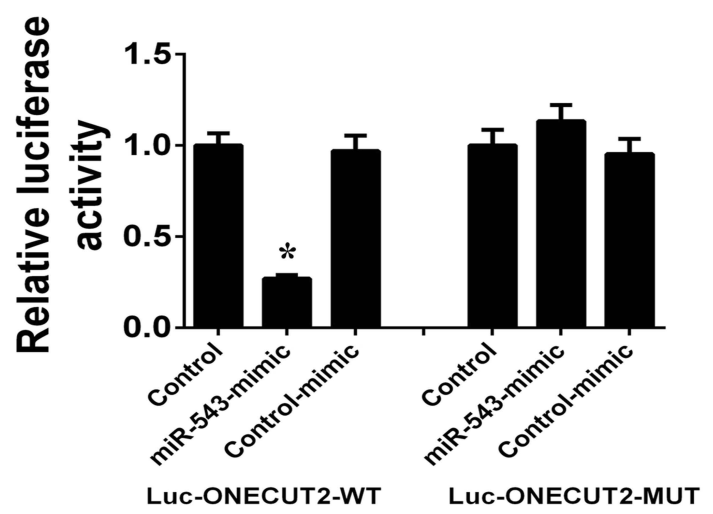

B
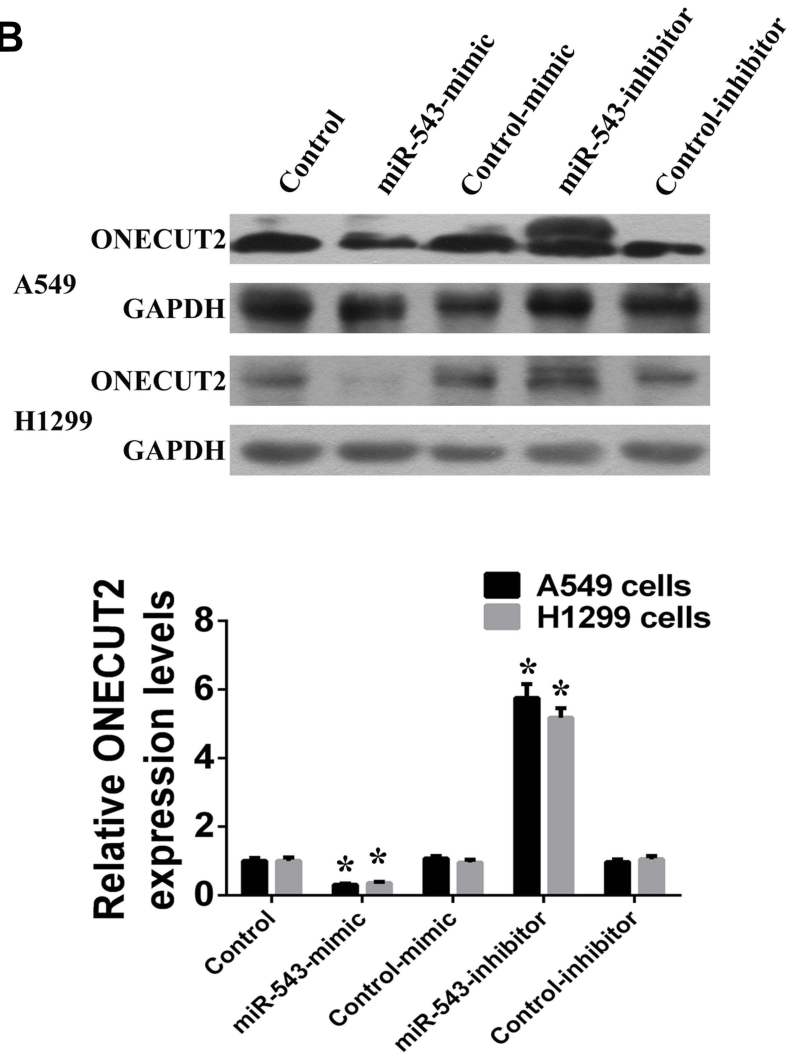

Figure 3 ONECUT2 was the direct target gene of miR-543. (A) The 3' untranslated region (3'UTR) of ONECUT2 contains the binding site for miR-543, including wild type and mutant type. (B) miR-543 regulated the protein and mRNA expression of ONECUT2 in A549 and HI299 cells. (C) Luciferase reporter assay was used to observe the binding and targeting effects of miR-543 on the $3^{\prime} U T R$ of ONECUT2. ( $* P<0.05$, error bar refers to SD).

study, it was shown that THUMPD3-AS1 was upregulated in NSCLC tissues and high expression of THUMPD3-AS1 was associated with TNM stages and relapse. Functional analysis found that THUMPD3-AS1 was involved in the regulation of cell proliferation and self-renewal in NSCLC cells. THUMPD3-AS1 is located in the genomic 3 p25.3 locus and can be transcribed into an IncRNA of 1951 nucleotides. Moreover, bioinformatics analysis showed that THUMPD3-AS1 contained binding sites for miR-543, and overexpression of THUMPD3-AS1 led to a decrease in miR-543, while knockdown of THUMPD3-AS1 led to an increase in miR-543. Therefore, it was found that THUMPD3-AS1 was associated with NSCLC and had the potential to regulate miR-543 as a ceRNA in NSCLC.

The miRNAs are endogenous non-coding small RNAs of about 18-25 nucleotides in length that are highly conserved in evolution and highly specific in tissues. $^{24}$ The miRNAs have post-transcriptional gene regulatory functions, and can degrade mRNA or inhibit mRNA translation by binding to the 3'UTR of the target gene mRNA. ${ }^{25}$ It is known that a number of miRNAs have been implicated in a wide variety of malignancies, and miR-543 is no exception. ${ }^{26-28}$ It has been found that miR-543 was greatly downregulated in glioma cell lines and tissues and was recognized as a tumor suppressor miRNA in glioma. ${ }^{29}$ Moreover, several findings demonstrated the tumor suppressor role of miR-543 in cervical cancer and breast cancer. ${ }^{30,31}$ In the present study, we confirmed that THUMPD3-AS1 regulated ONECUT2 as an endogenous sponge of miR-543 in NSCLC, suggesting that miR-543 also functioned as a tumor suppressor in NSCLC.

ONECUT2 is a transcription factor associated with development, and recent evidence has shown that aberrant expression of ONECUT2 in cancers is relevant in cancer progression. ${ }^{32-34}$ The expression of ONECUT2 has been found to be upregulated and drives tumor aggressiveness in ovarian cancer, prostate cancer and colorectal cancer. ${ }^{35-38}$ Moreover, it has been found that ONECUT2 was involved in the regulation of cancer stem cell-like features in breast cancer. ${ }^{39}$ However, the 
A

ONECUT2

A549

GAPDH

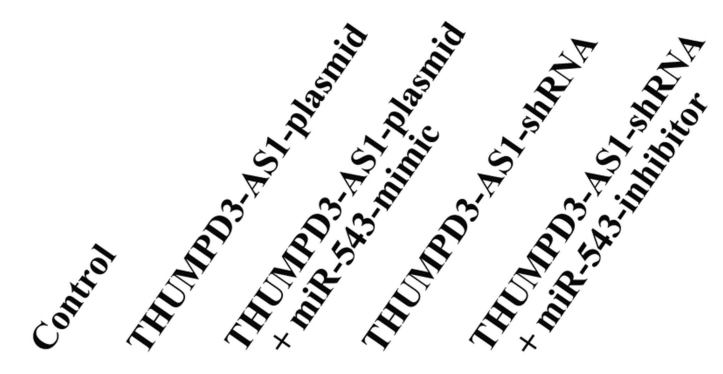

논

A549 cells

H1299 cells
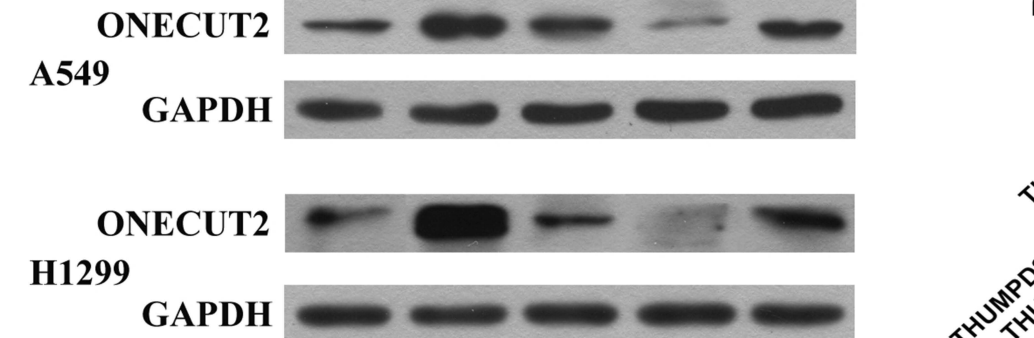

0
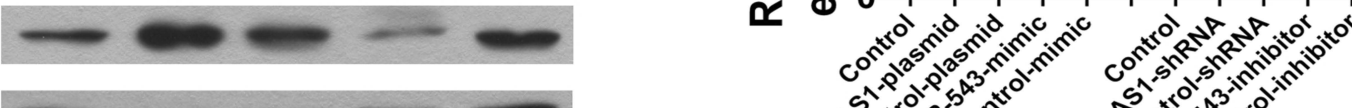

B

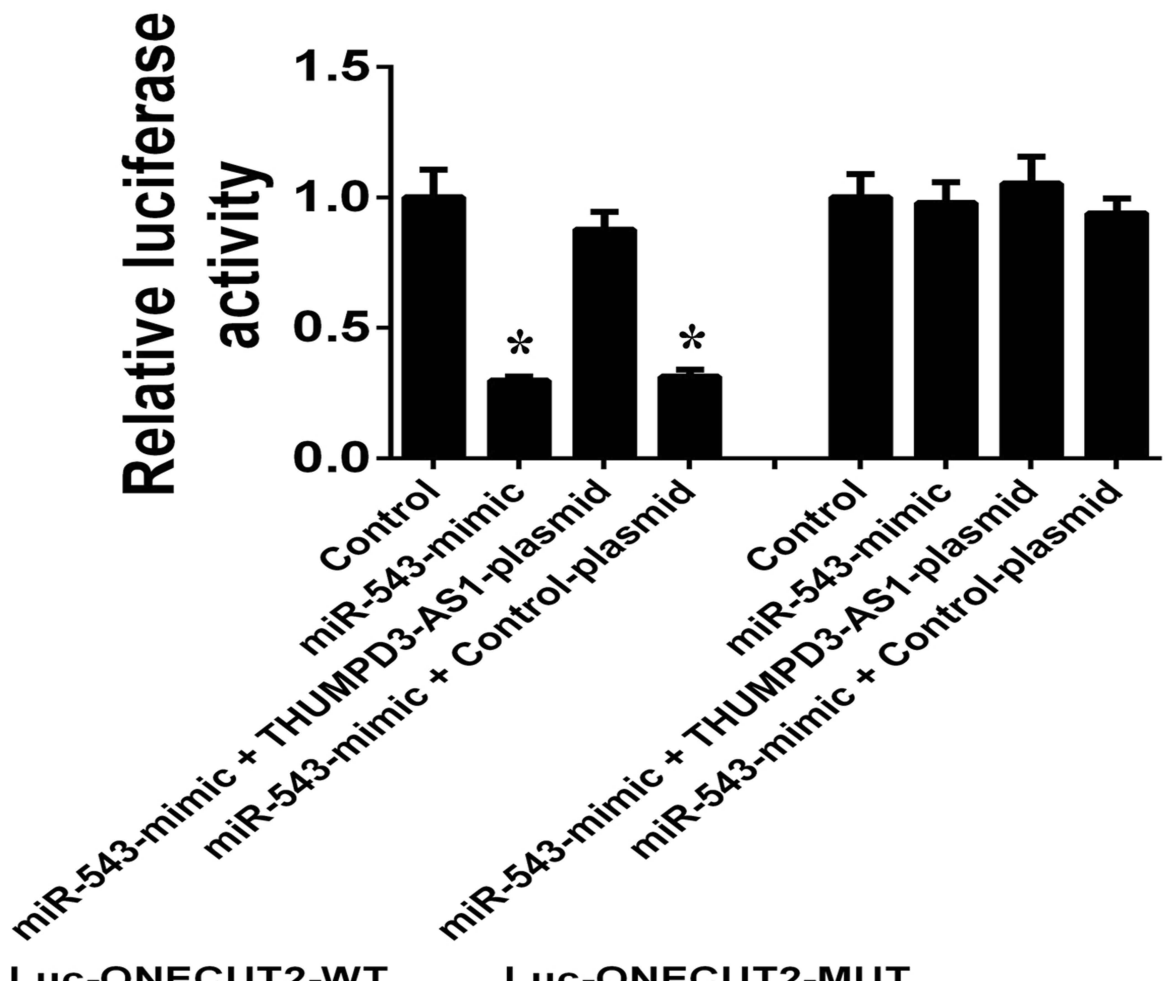

Figure 4 THUMPD3-ASI act as endogenous sponge of miR-543 to regulate ONECUT2 indirectly. (A) THUMPD3-ASI regulates the protein and mRNA expression of ONECUT2 through miR-543 in A549 and HI299 cells. (B) Luciferase reporter assay was used to confirm the regulating effects of THUMPD3-ASI on the 3'UTR of ONECUT2 through miR-543. (*P $<0.05$, error bar refers to $S D)$.

function of ONECUT2 in NSCLC has not yet been reported. In this study, it was demonstrated that THUMPD3-AS1 exhibited oncogene activity that promoted self-renewal of NSCLC cells through the regulation of ONECUT2, suggesting that ONECUT2 could promote stemness in NSCLC. 
A

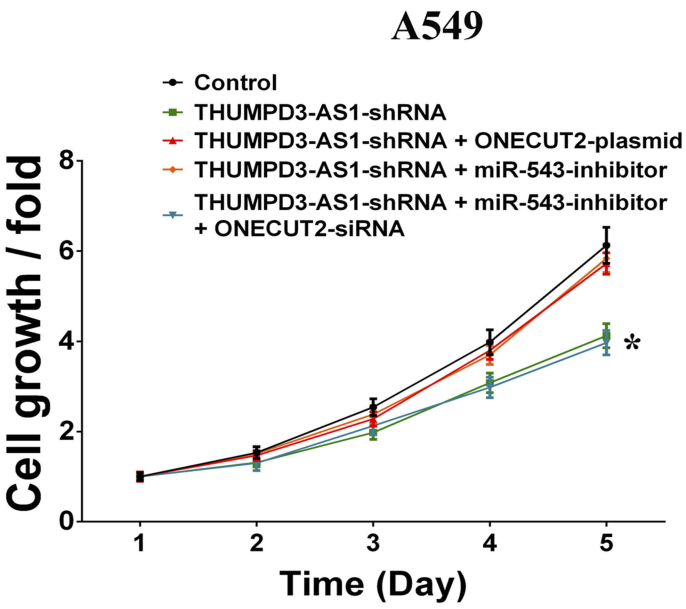

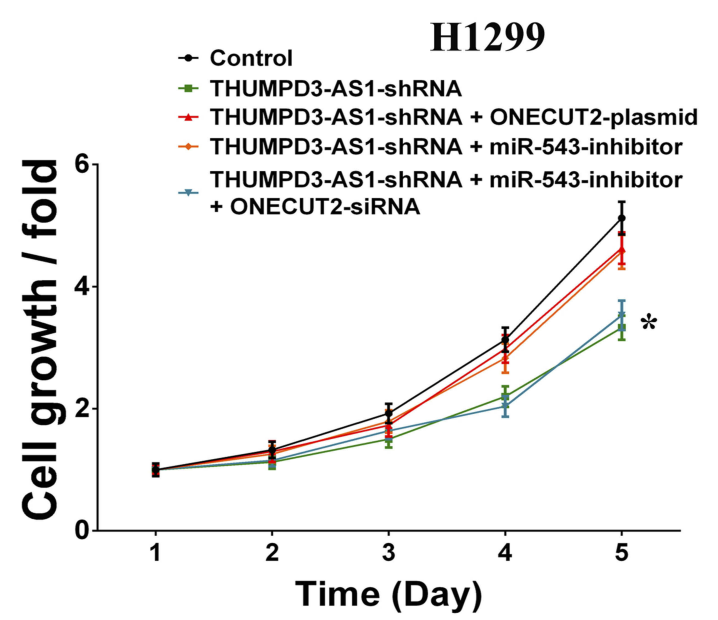

THUMPD3-AS1-shRNA

B

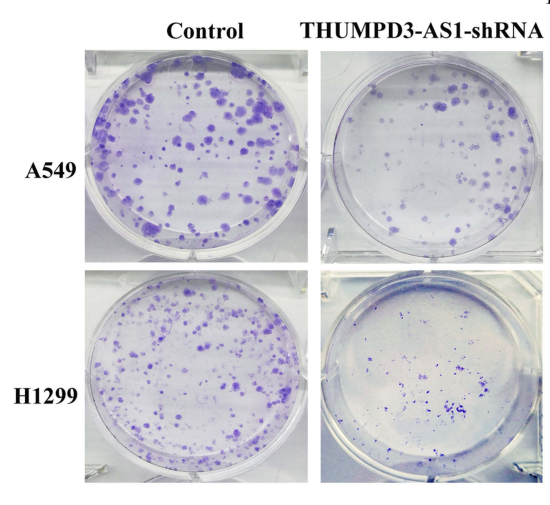

THUMPD3-AS1-shRNA THUMPD3-AS1-shRNA + miR-543-inhibitor
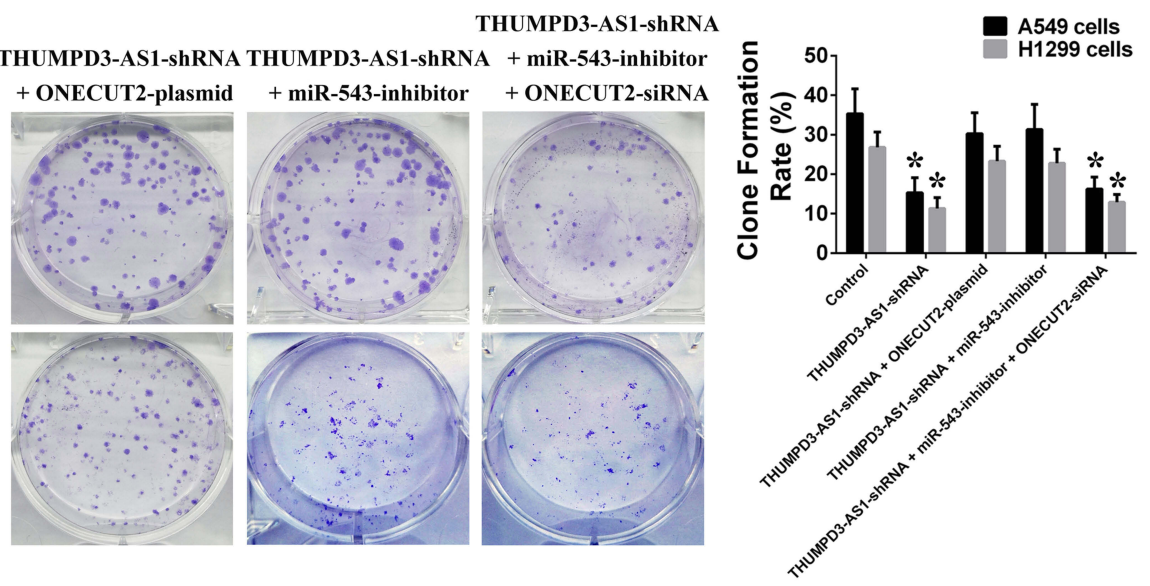

C

THUMPD3-AS1-shRNA THUMPD3-AS1-shRNA THUMPD3-AS1-shRNA + miR-543-inhibitor
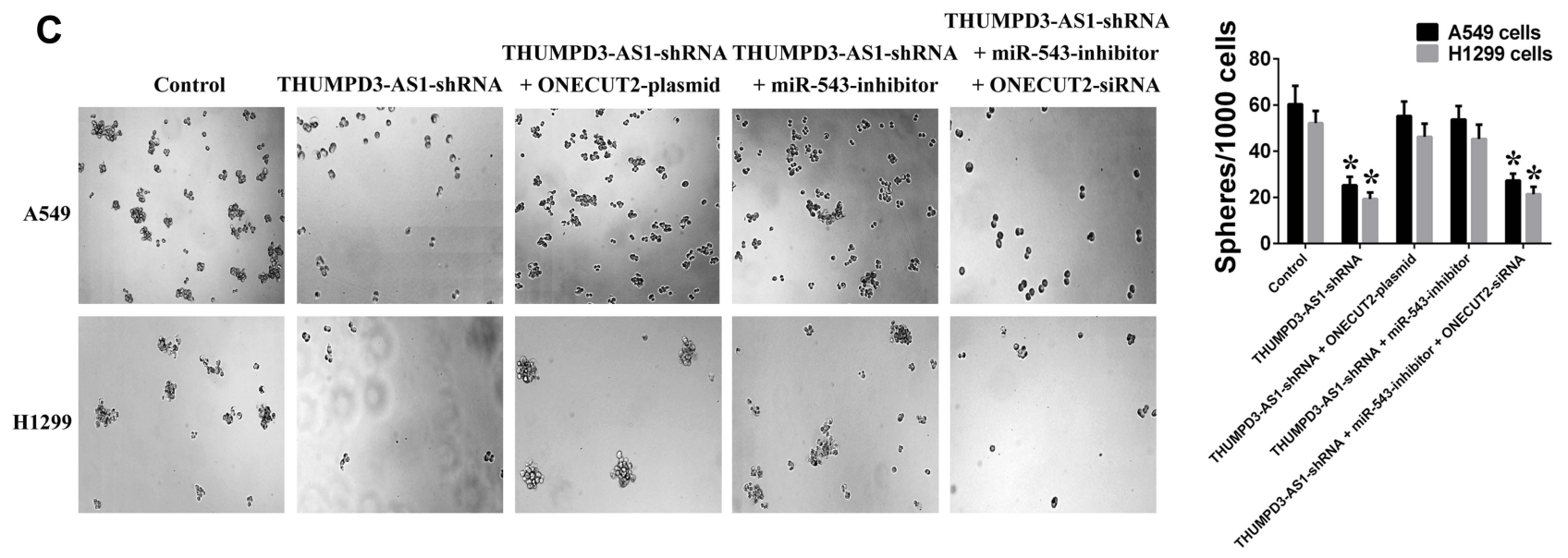

Figure 5 THUMPD3-ASI regulated cell proliferation and self-renewal by miR-543 and ONECUT2. (A) Individual or combined regulation of ONECUT2 and miR-543 influenced the regulation effect of THUMPD3-ASI on the cell growth in A549 and HI299 cells. (B) Individual or combined regulation of ONECUT2 and miR-543 influenced the regulation effect of THUMPD3-ASI on the colony-formation capability in A549 and HI299 cells. (C) Individual or combined regulation of ONECUT2 and miR-543 influenced the regulation effect of THUMPD3-ASI on the size and number of spheres in A549 and HI 299 cells. ( $* P<0.05$, error bar refers to SD).

\section{Conclusion}

This study extends the knowledge on the regulation of ONECUT2 at the post-transcription level by THUMPD3-
AS1 which can act as an endogenous sponge for miR-543, and the abnormal regulation of THUMPD3-AS1/miR-543/ ONECUT2 was involved in the regulation of self-renewal 


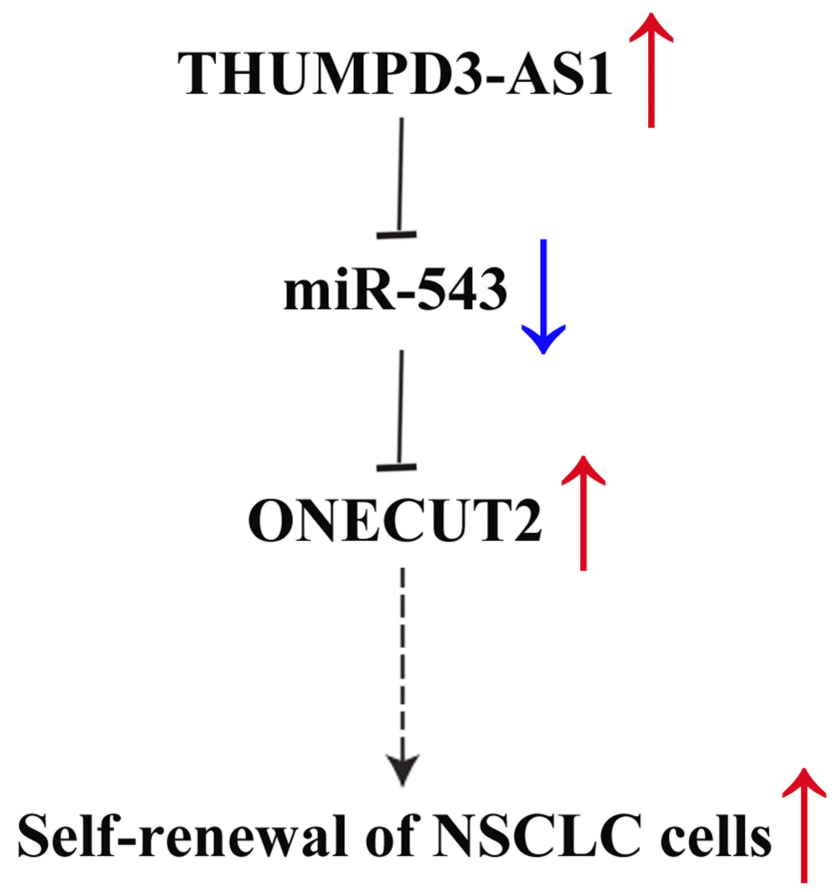

Figure 6 A summary diagram of THUMPD3-ASI, miR-543 and ONECUT2 func tion on self-renewal in NSCLC.

in NSCLC (Figure 6). Such novel molecular mechanisms which sustain NSCLC provide a new biomarker and therapy target for NSCLC and provide a molecular basis for tumor progression.

\section{Ethics Approval And Consent To Participate}

Written informed consent was obtained from all patients. This study was approved by the Ethics Committee of Sun Yat-sen University Cancer Center (approval number: GZR 2018-120).

\section{Acknowledgement}

This study was supported by grants from the National Natural Science Foundation of China (81871986).

\section{Disclosure}

The authors report no conflicts of interest in this work.

\section{References}

1. Siegel RL, Miller KD, Jemal A. Cancer statistics, 2018. CA Cancer J Clin. 2015;68:7-30. doi:10.3322/caac.21442

2. Chen W, Zheng R, Baade PD, et al. Cancer statistics in China, 2015. CA Cancer J Clin. 2016;66:115-132. doi:10.3322/caac.21338

3. Altorki NK, Markowitz GJ, Gao D, et al. The lung microenvironment: an important regulator of tumour growth and metastasis. Nat Rev Cancer. 2019;19(1):9-31.
4. Vargas AJ, Harris CC. Biomarker development in the precision medicine era: lung cancer as a case study. Nat Rev Cancer. 2016;16 (8):525-537. doi:10.1038/nrc.2016.56

5. Abbosh C, Birkbak NJ, Swanton C. Early stage NSCLC - challenges to implementing ctDNA-based screening and MRD detection. Nat Rev Clin Oncol. 2018;15(9):577-586. doi:10.1038/s41571-018-0058-3

6. Matsui M, Corey DR. Non-coding RNAs as drug targets. Nat Rev Drug Discov. 2017;16(3):167-179. doi:10.1038/nrd.2016.117

7. Sun Q, Hao Q, Prasanth KV. Nuclear long noncoding RNAs: key regulators of gene expression. Trends Genet. 2018;34(2):142-157. doi:10.1016/j.tig.2017.11.005

8. Greco S, Gaetano C, Martelli F. Long noncoding competing endogenous RNA networks in age-associated cardiovascular diseases. Int J Mol Sci. 2019;20(12):E3079. doi:10.3390/ijms20123079

9. Yao RW, Wang Y, Chen LL. Cellular functions of long noncoding RNAs. Nat Cell Biol. 2019;21(5):542-551. doi:10.1038/s41556-0190311-8

10. Quinn JJ, Chang HY. Unique features of long non-coding RNA biogenesis and function. Nat Rev Genet. 2016;17(1):47-62. doi:10.1038/nrg.2015.10

11. Fatica A, Bozzoni I. Long non-coding RNAs: new players in cell differentiation and development. Nat Rev Genet. 2014;15(1):7-21. doi: $10.1038 / \mathrm{nrg} 3606$

12. Wang J, Zhang X, Chen W, et al. Regulatory roles of long noncoding RNAs implicated in cancer hallmarks. Int J Cancer. 2019. doi:10.1002/ijc.32277

13. Xie Y, Dang W, Zhang S, et al. The role of exosomal noncoding RNAs in cancer. Mol Cancer. 2019;18(1):37. doi:10.1186/s12943019-0984-4

14. Osielska MA, Jagodziński PP. Long non-coding RNA as potential biomarkers in non-small-cell lung cancer: what do we know so far? Biomed Pharmacother. 2018;101:322-333. doi:10.1016/j.biopha.2018.02.099

15. Ricciuti B, Mencaroni C, Paglialunga L, et al. Long noncoding RNAs: new insights into non-small cell lung cancer biology, diagnosis and therapy. Med Oncol. 2016;33(2):18. doi:10.1007/s12032016-0731-2

16. Li DS, Ainiwaer JL, Sheyhiding I, et al. Identification of key long non-coding RNAs as competing endogenous RNAs for miRNAmRNA in lung adenocarcinoma. Eur Rev Med Pharmacol Sci. 2016;20(11):2285-2295.

17. Ebert MS, Sharp PA. Emerging roles for natural microRNA sponges. Curr Biol. 2010;20:R858-R861. doi:10.1016/j.cub.2010.08.052

18. Thomson DW, Dinger ME. Endogenous microRNA sponges: evidence and controversy. Nat Rev Genet. 2016;17(5):272-283. doi:10. 1038/nrg.2016.20

19. Abdollahzadeh R, Daraei A, Mansoori Y, et al. Competing endogenous RNA (ceRNA) cross talk and language in ceRNA regulatory networks: A new look at hallmarks of breast cancer. J Cell Physiol. 2019;234(7):10080-10100. doi:10.1002/jcp.v234.7

20. Tay Y, Rinn J, Pandolfi PP. The multilayered complexity of ceRNA crosstalk and competition. Nature. 2014;505(7483):344-352. doi:10. 1038/nature12986

21. Qi X, Zhang DH, Wu N, et al. ceRNA in cancer: possible functions and clinical implications. $J$ Med Genet. 2015;52(10):710-718. doi:10.1136/jmedgenet-2015-103334

22. Fang L, Wu S, Zhu X, et al. MYEOV functions as an amplified competing endogenous RNA in promoting metastasis by activating TGF- $\beta$ pathway in NSCLC. Oncogene. 2019;38(6):896-912. doi:10. 1038/s41388-018-0484-9

23. Alam M, Ahmad R, Rajabi $\mathrm{H}$, et al. MUC1-C induces the LIN28B $\rightarrow$ LET-7 $\rightarrow$ HMGA2 axis to regulate self-renewal in NSCLC. Mol Cancer Res. 2015;13(3):449-460. doi:10.1158/15417786.MCR-14-0363

24. Treiber T, Treiber N, Meister G. Regulation of microRNA biogenesis and its crosstalk with other cellular pathways. Nat Rev Mol Cell Biol. 2019;20(1):5-20. doi:10.1038/s41580-018-0059-1 
25. Gebert LFR, MacRae IJ. Regulation of microRNA function in animals. Nat Rev Mol Cell Biol. 2019;20(1):21-37.

26. Rupaimoole R, Slack FJ. MicroRNA therapeutics: towards a new era for the management of cancer and other diseases. Nat Rev Drug Discov. 2017;16(3):203-222. doi:10.1038/nrd.2016.246

27. Di Leva G, Garofalo M, Croce CM. MicroRNAs in cancer. Annu Rev Pathol. 2014;9:287-314. doi:10.1146/annurev-pathol-012513104715

28. Lu J, Zhan Y, Feng J, et al. MicroRNAs associated with therapy of non-small cell lung cancer. Int J Biol Sci. 2018;14(4):390-397. doi: $10.7150 /$ ijbs. 22243

29. Xu L, Yu J, Wang Z, et al. miR-543 functions as a tumor suppressor in glioma in vitro and in vivo. Oncol Rep. 2017;38(2):725-734. doi:10.3892/or.2017.5712

30. Liu X, Gan L, Zhang J. miR-543 inhibites cervical cancer growth and metastasis by targeting TRPM7. Chem Biol Interact. 2019;302:8392. doi:10.1016/j.cbi.2019.01.036

31. Chen P, Xu W, Luo Y, et al. MicroRNA 543 suppresses breast cancer cell proliferation, blocks cell cycle and induces cell apoptosis via direct targeting of ERK/MAPK. Onco Targets Ther. 2017;10:14231431. doi:10.2147/OTT

32. Sapkota $\mathrm{D}$, Chintala $\mathrm{H}, \mathrm{Wu}$ F, et al. Onecut1 and Onecut2 redundantly regulate early retinal cell fates during development. Proc Natl Acad Sci USA. 2014;111(39):E4086-E4095. doi:10.1073/pnas.1405 354111
33. Klimova L, Antosova B, Kuzelova A, et al. Onecut1 and Onecut2 transcription factors operate downstream of Pax6 to regulate horizontal cell development. Dev Biol. 2015;402(1):48-60. doi:10.1016/j. ydbio.2015.02.023

34. Wu Y, Jiang G, Zhang N, et al. HOXA9, PCDH17, POU4F2, and ONECUT2 as a urinary biomarker combination for the detection of bladder cancer in chinese patients with hematuria. Eur Urol Focus. 2018;S2405-S4569(18):30289-X.

35. Lu T, Wu B, Yu Y, et al. Blockade of ONECUT2 expression in ovarian cancer inhibited tumor cell proliferation, migration, invasion and angiogenesis. Cancer Sci. 2018;109(7):2221-2234. doi:10.1111/cas.13633

36. Guo H, Ci X, Ahmed M, et al. ONECUT2 is a driver of neuroendocrine prostate cancer. Nat Commun. 2019;10(1):278. doi:10.1038/ s41467-018-08133-6

37. Rotinen M, You S, Yang J, et al. ONECUT2 is a targetable master regulator of lethal prostate cancer that suppresses the androgen axis. Nat Med. 2018;24(12):1887-1898. doi:10.1038/s41591-018-0241-1

38. Sun Y, Shen S, Liu X, et al. MiR-429 inhibits cells growth and invasion and regulates EMT-related marker genes by targeting onecut2 in colorectal carcinoma. Mol Cell Biochem. 2014;390(1-2):1930. doi:10.1007/s11010-013-1950-x

39. Shen M, Dong C, Ruan X, et al. Chemotherapy-induced extracellular vesicle mirnas promote breast cancer stemness by targeting ONECUT2. Cancer Res. 2019;79(14):3608-3621. doi:10.1158/000 8-5472.CAN-18-4055

\section{Publish your work in this journal}

OncoTargets and Therapy is an international, peer-reviewed, open access journal focusing on the pathological basis of all cancers, potential targets for therapy and treatment protocols employed to improve the management of cancer patients. The journal also focuses on the impact of management programs and new therapeutic agents and protocols on patient perspectives such as quality of life, adherence and satisfaction. The manuscript management system is completely online and includes a very quick and fair peer-review system, which is all easy to use. Visit http://www.dovepress.com/ testimonials.php to read real quotes from published authors. 